\title{
Identification and removal of non-acoustic noise in towed array sonar using F-K transform for enhanced torpedo detection
}

\author{
Jomon George, Baiju M Nair, and T. Santhanakrishnan
}

\begin{abstract}
Low frequency passive towed array sonar is an essential component in a torpedo detection system for surface ships. Compact towed arrays are used for torpedo detection and they will be towed at higher towing speeds compared to conventional towed array sonars used for surveillance. Presence of non-acoustic noise in towed array sensors at higher towing speeds degrades torpedo detection capability at lower frequencies. High wavenumber mechanical vibrations are induced in the array by vortex shedding associated with hydrodynamic flow over the array body and cable scope. These vibrations are known to couple into the hydrophone array as nonacoustic noise sources and can impair acoustic detection performance, particularly in the forward end fire direction. Lengthy mechanical vibration isolation modules can isolate vibration induced noise in towed arrays, but this is not recommended in a towed array which is towed at high speeds as it will increase the drag and system complexity. An algorithm for decomposing acoustic and non-acoustic components of signals received at sensor level using well known frequency-wavenumber transform (F-K transform) is presented here. Frequency-wavenumber diagrams can be used for differentiating between acoustic and non-acoustic signals. An area of V shape is identified within the F-K spectrum where acoustic energy is confined. Energy outside this V will highlight non-acoustic energy. Enhanced simultaneous spatio-temporal and spatio-amplitude detection is possible with this algorithm. Performance of this algorithm is validated through simulation and experimental data.
\end{abstract}

Keywords- Towed Array Sonar, Torpedo defence system, Acoustic signal processing, F-K Transform.

\section{INTRODUCTION}

Low frequency Towed Array Sonars (TAS) operating in passive mode are widely used in surface ships for long range detection and localisation of fast-moving underwater targets like torpedoes. Torpedo detection is considered as one of the most important tasks in Anti-Submarine Warfare (ASW) [1, 2]. Towed array sonars are preferred over classical Hull mounted Sonars (HMS) for torpedo detection [3, 4]. Operational advantages of towed array sonars are listed below.

- Long arrays with large acoustic aperture is feasible with TAS, which is essential for low frequency operation for long range detection,

- Towed arrays can be deployed at desired depth for optimum in-layer and below layer detection by controlling the cable payout length or by controlling the speed of the towing platform,

- Towed arrays can be deployed far behind the towing ship by adjusting the cable payout to reduce the impact of the platform noise level on the hydrophones in the array.
Typical configuration of towed array sonar is shown in Fig. 1. A linear array of hydrophones in the form of a towed array is deployed behind the ship for sensing the radiated acoustic signature of the target $[5,6]$. In towed arrays, hydrophones are uniformly spaced at $\lambda / 2$, where $\lambda$ is the wavelength corresponds to the maximum operating frequency of the array. Hydrophone outputs after pre-amplification, filtering and digitization are sent to onboard processing units in Ethernet format for target detection, localisation and classification [7]. Hydraulic or electric winch is used for the deployment and retrieval of the towed array. Electro mechanical tow cable is the umbilical cord between the towed array and onboard processing units. It is used for power transmission from onboard units to towed array and data interface between towed array and onboard units. Towed array hydrophone data in Ethernet format is transmitted through tow cable using optical medium. Tail rope is used as an aft stabilization module to reduce array snaking effect.

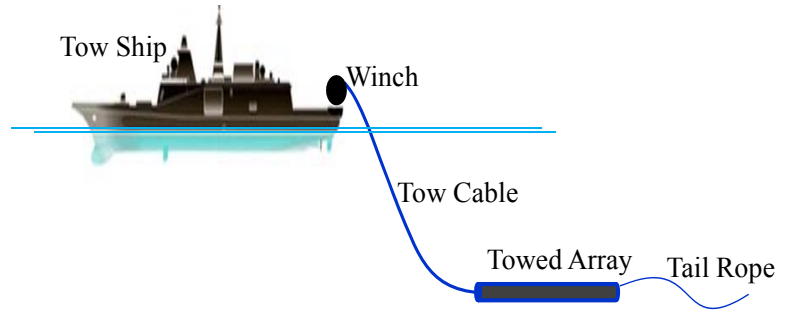

Fig. 1. Towed array sonar configuration

Early detection of an attacking torpedo is essential for enhancing the probability of escape by employing torpedo countermeasures [8]. Countermeasures include platform maneuvering, by deploying acoustic decoys and by deploying counter attack on an attacking torpedo $[9,10]$.

Towed arrays are generally towed at higher towing speeds for a torpedo defence system compared to a towed array sonar used for surveillance [11]. Presence of non-acoustic noise at the sensor output at higher towing speeds degrades the detection performance. Lengthy Vibration Isolation Modules (VIM) can be used at the front and back end of the array to reduce vibration induced noise at the sensor [12]. Usage of longer VIM is not recommended for torpedo defence systems as it will increase drag during highspeed towing and it will also increase the complexity of the system. Signal processing technics can be effectively utilised for identification and removal of non-acoustic noise from towed array sensor output. 
Well known Frequency Wavenumber transform

(F-K

Transform) is proposed here for identification and separation of acoustic and non-acoustic components from sensor outputs. Frequency-wavenumber (F-K) analysis exploits the velocity differences among wave fronts received at the sensor and provides a tool for analysing relative power of acoustic and non-acoustic noise contributions on the towed array [13 - 15].

The paper is organised as follows. In Section 2 detailed theoretical overview of flow noise is given. Basics of F-K transform is given in Section-3. F-K transform analysis of towed array experiment data is presented in Section-4. F-K transform for enhanced torpedo detection is presented in Section-5. This led to conclusions in Section 6.

\section{FLOW INDUCED NOISE ON A TOWED ARRAY}

Water flow along the towed array creates turbulence induced noise on hydrophones, especially at higher towing speeds. Flow induced excitation of vibrations in the array and the effect of the turbulent boundary layer near the hose wall creates turbulence-induced noise on the hydrophones [16]. These noises are not true sound, but do influence hydrophone output. These types of noise generally termed as non-acoustic noise.

Tow cable strumming and towed array tail wagging causes vibrating force at the towed array which induces breathing waves in the fluid filled hose, extensional waves in the hose wall, and resonances and other very long waves within the array [17]. These array vibrations will contribute significantly to vibration noise, especially towed arrays operating at low frequencies in passive mode. The vibrational energy propagates along the array in longitudinal waves in hose wall and strength members. An object that is attached to the hose wall and/or the strength members is forced into vibration. These objects absorb energy of the breathing wave frequency to transmit this energy again in a much wider spectrum [18, 19]. Lengthy forward and aft VIM can isolate vibration induced noise upto certain level at moderate towing speeds.

Along with vibrations of the array, the direct transmission of the pressure fluctuations, of the turbulent boundary layer along the acoustic modules, into the fluid filled tube also generates flow noise in the towed array. VIM is not a solution for isolating turbulent pressure fluctuations. Properly designed hose wall can filter this pressure fluctuations in a minimal way $[20,21]$.

\section{F-K TRANSFORM}

Beamformer is used with an array of sensors to determine the direction in which the acoustic signal source is located. The sensor element outputs are combined to form multiple narrow beams, each of which look in a single direction and are insensitive to sound incident from neighboring directions [22, 23]. Adaptive beam forming techniques are also used to enhance the detection performance by reducing beam width and side lobe levels [24]. Conventional delay-sum beamformer depicts the scenario as bearing vs. amplitude and it lacks the information on the temporal variation of the signal. For certain applications like torpedo detection, where a spatiotemporal view is required, frequency domain beamformer is employed.

F-K transform is used to generate simultaneous spatioamplitude and spatio-temporal view for a linear array. F-K transform is derived from two-dimensional spectrum of a line array. This method is computationally less expensive and is faster than the conventional frequency domain beamformer since only the Fast Fourier Transform (FFT) operations are involved. The conventional spatio-temporal view can be generated from the F-K transform by a simple look-up table method. Also the conventional bearing vs. amplitude view can be generated from the F-K transform by line integration.

The F-K transformation is in-principle a two-dimensional Fourier transformation. Corresponding to the transformation of the time-axis to the frequency domain, the $x$-axis is transformed to the wavenumber domain [25]. The Wavenumber $K$ indicates the number of wavelengths per meter along the horizontal axis, for waves which propagate horizontally, the transformation returns the actual wavenumber. For waves that do not propagate horizontally, the horizontal component of the wave is transformed.

The plotting of a dataset in F-K domain is called an F-K spectrum. Analogous to the frequency spectrum for the onedimensional transformation from time to frequency. The signals are separated and plotted as function of the frequency and wave number.

\section{A. The frequency-wavenumber grid}

The frequency wavenumber analysis is a wave filter, which filters both in frequency and spatial domain simultaneously [26,15]. Consider a linear array of $M$ sensors spaced at $d$ as shown in Fig. 2. Given an incident plane wave of frequency $F$ and arrival angle $\theta$ with velocity $c$, its spatial frequency, or wavenumber $K$, as seen along the axis of the array is defined as

$$
K=(2 \pi f d / c) \sin (\theta) .
$$

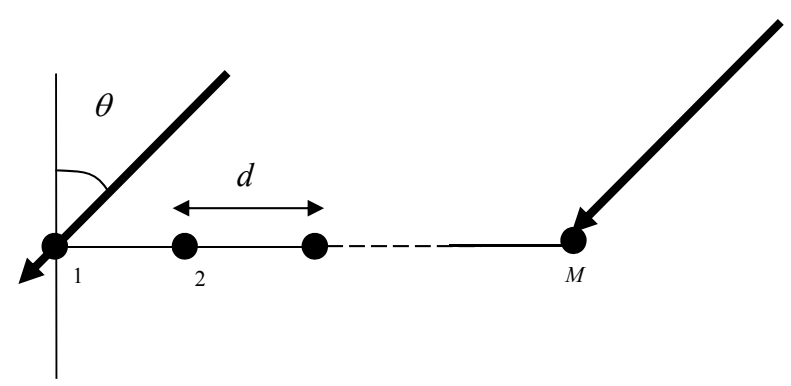

Fig. 2. Linear array of $N$ sensors with an acoustic source at an angle $\theta$

Let $\left\{x_{m}(i)\right\}$ denote the time series from the $m^{\text {th }}$ hydrophone, where $0 \leq m \leq M-1$ and $i$ is the time index of the sampled data. The time series from each sensor is segmented into blocks, and the Discrete Fourier Transform (DFT) coefficients $X_{m}(k)$ are computed, where $k$ is the frequency index. Select 
suitable window function $w_{m}$ for computing frequency wavenumber coefficients as given in Eq. 2.

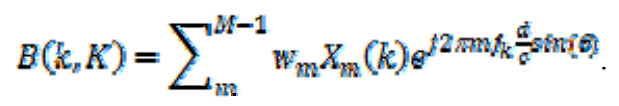

Here, $K$ is the wavenumber, $f_{k}=k f_{s} / N$ for sampling frequency $f_{s}$, FFT length $N$ and $w_{m}$ is the suitable window function. A plot of $|B(k, K)|^{2}$ with wavenumber as $x$-axis and frequency as $y$-axis will generate typical $\mathrm{V}$-shaped frequency wavenumber grid as shown in Fig. 3 for frequency upto designed frequency $f_{d}$ of the array. The acoustic energy is confined within the V-shape of the plot. The area outside the shaded region of the grid is the non-acoustic region, in which no waterborne acoustic signal can lie, and the area within the dotted lines is the region in which spatial aliasing cannot occur. The fundamental assumption behind the statement is that the acoustic noise travels with a speed around $1500 \mathrm{~m} / \mathrm{s}$ in water, while non-acoustic noise travel at different speed.

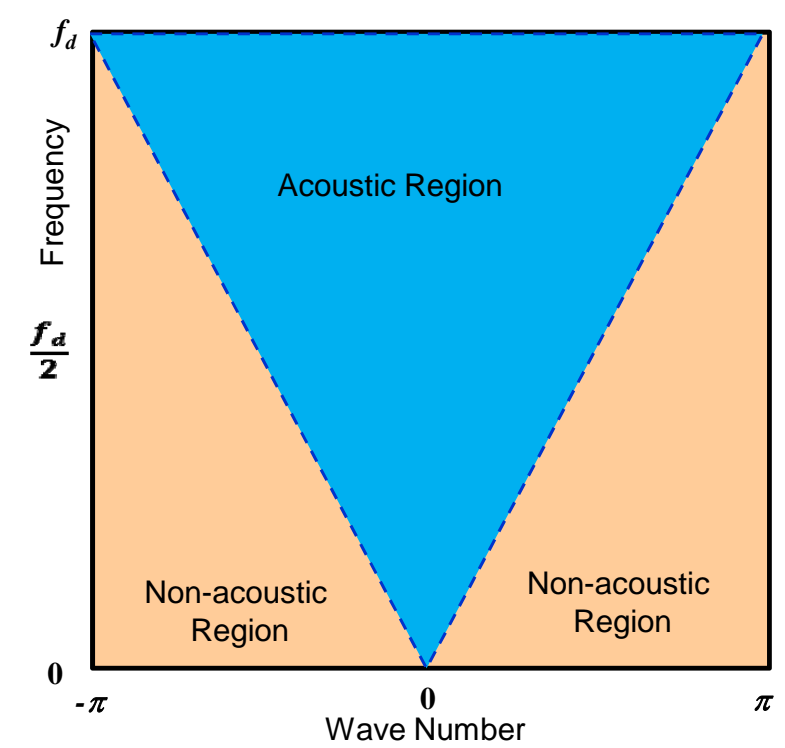

Fig. 3. Frequency-wavenumber grid

\section{EXPERIMENT TO MEASURE NON-ACOUSTIC NOISE}

Presence of non-acoustic noise in towed arrays and its effect due to towing speed is measured during towing trial and the results are presented here. The experimental data was collected during sea trials using a towed array of length $20 \mathrm{~m}$ with 32 hydrophones spaced for $2-\mathrm{kHz}$ operating band. $10 \mathrm{~m}$ tail rope was connected at the aft end of the array and the array was towed $500 \mathrm{~m}$ behind the ship. No VIM was used for the trials. The trial was conducted at sea state 1-2 and the depth of the sea at trial area was more than $1000 \mathrm{~m}$. The data from the hydrophones were collected and analysed for different towing speeds. F-K transform plot for 8 knots towing speed is shown in Fig. 4(a). F-K spectrum in 2D and 3D format is shown at left and right sides, respectively. Similar plot for 16 knots towing speed is shown in Fig. 4(b). Presence of stronger nonacoustic low frequency components is evident at higher towing speed.
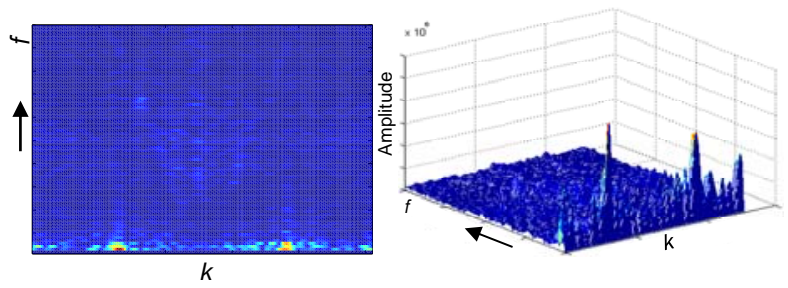

(a)
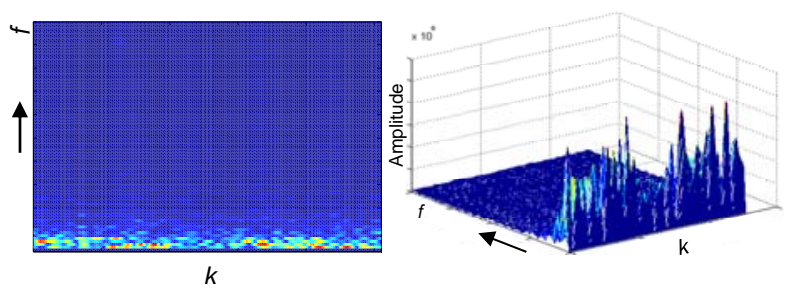

(b)
Fig. 4. F-K transform plot in $2 \mathrm{D}$ and $3 \mathrm{D}$ format for (a) 8 knots and (b) 16 knots towing speed

Presence of non-acoustic noise in towed array sonars vary with respect to towing speed. Table-1 shows the percentage of non-acoustic energy present in the sensor output in the desired operating band for different towing speeds. Presence of nonacoustic noise will degrade the detection performance of the sonar. This is a serious problem in torpedo defence system as these arrays are towed at higher towing speeds. Identification and removal of non-acoustic noise is an essential requirement for improved performance of torpedo defence systems.

Table 1. Non-acoustic noise energy vs. towing speed

\begin{tabular}{|c|c|c|}
\hline NO & $\begin{array}{c}\text { TOWING } \\
\text { SPEED } \\
\text { (Knots) }\end{array}$ & $\begin{array}{c}\text { AVERAGE } \\
\text { PERCENTAGE OF NON- } \\
\text { ACOUSTIC NOISE } \\
\text { ENERGY IN THE } \\
\text { DESIRED OPERATING } \\
\text { BAND }\end{array}$ \\
\hline 1 & 6 & $8 \%$ \\
\hline 2 & 8 & $12 \%$ \\
\hline 3 & 12 & $18 \%$ \\
\hline 4 & 16 & $28 \%$ \\
\hline 5 & 18 & $40 \%$ \\
\hline
\end{tabular}

\section{F-K TRANSFORM FOR ENHANCED TORPEDO DETECTION}

Spatio and temporal information of acoustic targets can be extracted from F-K spectrum simultaneously though the process shown in Fig. 5. This process can generate spatioamplitude and spatio-temporal plot without the influence of non-acoustic noise. This will enhance target detection performance. 


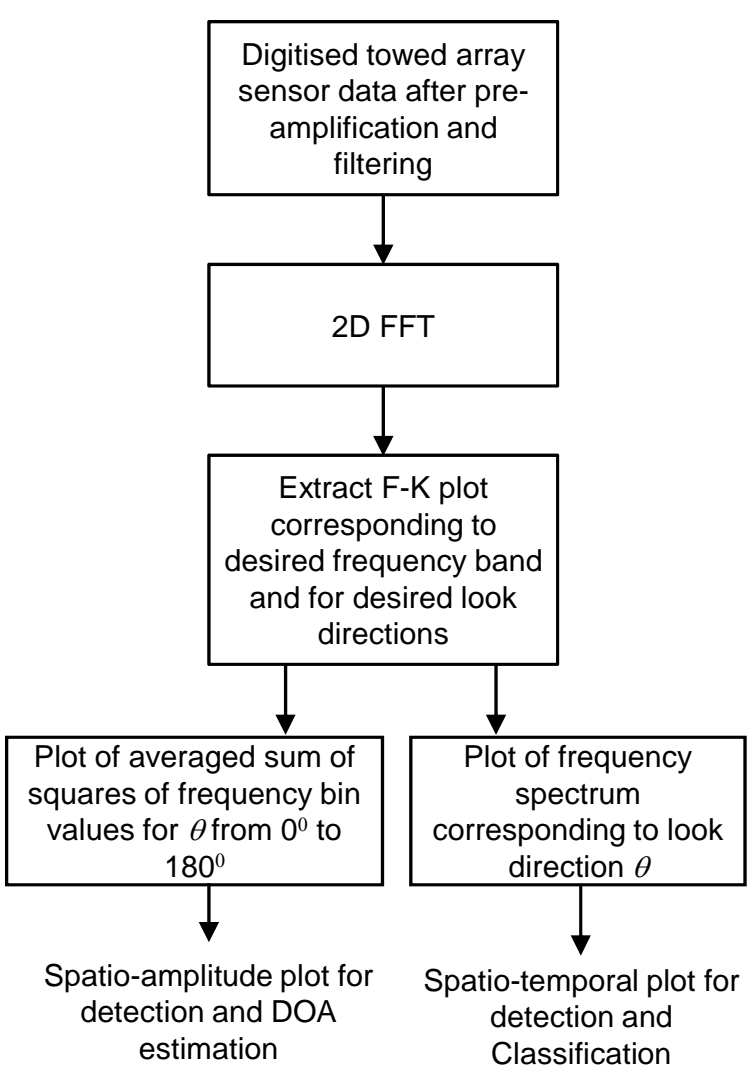

Fig. 5. Extraction of Spatio and temporal information of targets using F-K spectrum

Plots of spatio and temporal information extracted from F-K spectrum is shown in Fig. 6. Fig. 6(a) shows F-K spectrum of a linear array receiving signals from two acoustic targets located at $\theta_{1}$ and $\theta_{2}$ with respect to the linear array. Fig. 6(b) indicates the spatio-amplitude view, which represents the target bearing vs. acoustic energy amplitude view. This view after averaging is used for target detection and Direction Of Arrival (DOA) estimation. Spatio-amplitude view is generated by presenting the amplitude of the energy vs. look direction $\theta$, starting from $0^{0}$ to $180^{\circ}$ at sufficient resolution of $\theta$ where, $0^{0}$ corresponding to fore end of the array and $180^{\circ}$ corresponding to aft end of the array. Energy at a look direction $\theta$ is computed by integrating the energy from all frequency bins in the selected look direction $\theta$. FrequencyAmplitude view for a target at $\theta_{1}$ is shown in Fig. 6(c). Target spectral information is an essential input required for target classification. This view is generated by extracting the amplitude of the frequency bins corresponding to the look direction $\theta_{1}$. Panoramic spatio-temporal view also can be generated from F-K spectrum for early detection of targets with strong tonal components. This view is generated by presenting spectral components vs. look direction $\theta$, starting from $0^{0}$ to $180^{\circ}$ at sufficient resolution of $\theta$.

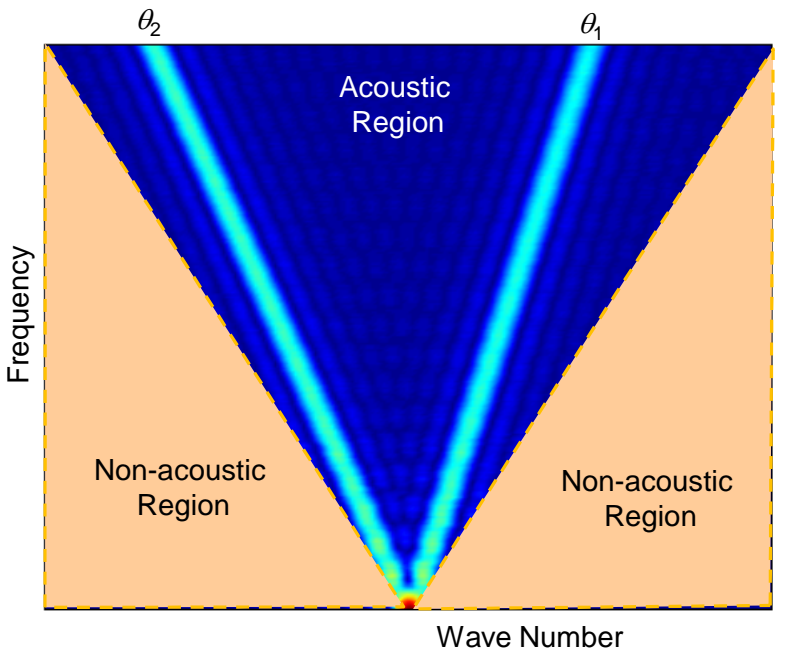

(a)

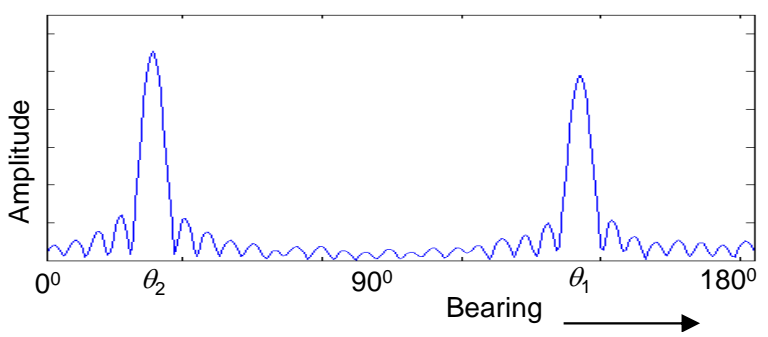

(b)

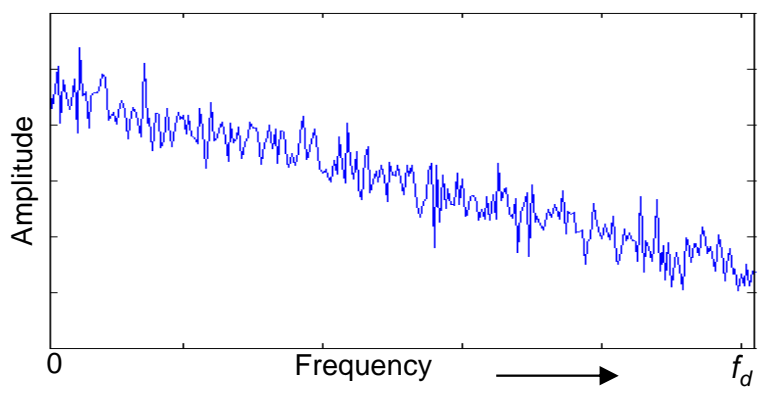

(c)

Fig. 6. Plots derived from F-K transform. (a) F-K Spectrum, (b) Spatio-amplitude plot, (c) Frequency-Amplitude plot for target at $\theta_{1}$

Bearing Time Record (BTR) can be plotted using spatioamplitude output computed at regular time intervals as shown in the bottom graph in Fig 7. In BTR display, $x$-axis represents the bearing and $y$-axis represents time history. Normalised amplitude is plotted in the BTR display [27]. The current time is marked on top of the display and the history is on the bottom. Target energy is marked as intensity levels on the display. Top graph indicates amplitude vs. bearing plot for the latest update. 


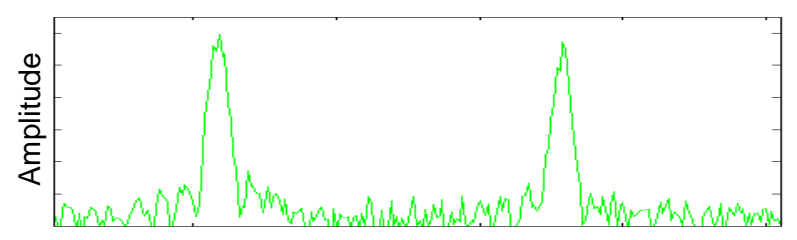

$0^{0}$

Bearing
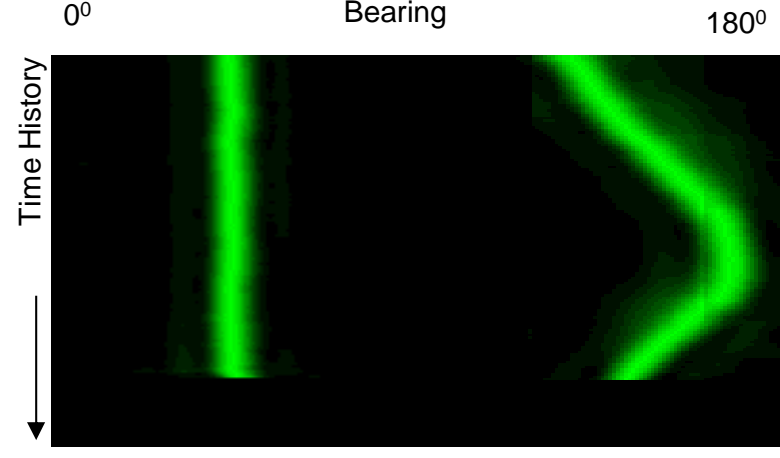

Fig. 7. BTR plot computed from B-K spectrum

\section{A. Hough Transform for automatic target detection.}

Automatic target detection is an important requirement for a torpedo detection system. Hough Transform (HT) can be used along with F-K spectrum for automatic target detection. HT is a feature extraction technique used for detecting lines from an image [28, 29]. For automatic contact detection, acoustic region of the B-K spectrum or $N$ recent updates of BTR plot can be used as an input matrix to the HT. A threshold is applied on this Matrix to identify signal energy above probable noise levels. Map all the points above this threshold in the Hough plane using the equation

$$
\rho=x \cos (\phi)+y \sin (\phi),
$$

where $\phi$ is the angle of the line from the origin orthogonal to the line and $\rho$ is the perpendicular distance from the origin to the line as shown in Fig 8. After mapping all the points above the primary threshold, a secondary threshold is applied to get the polar parameters ( $\rho$ and $\phi$ ) of the lines detected. After mapping $\rho$ to bearing axis, estimate target bearing $\theta$ and report as probable target bearing. Spectral information is extracted for this direction and initiate action for torpedo classification.

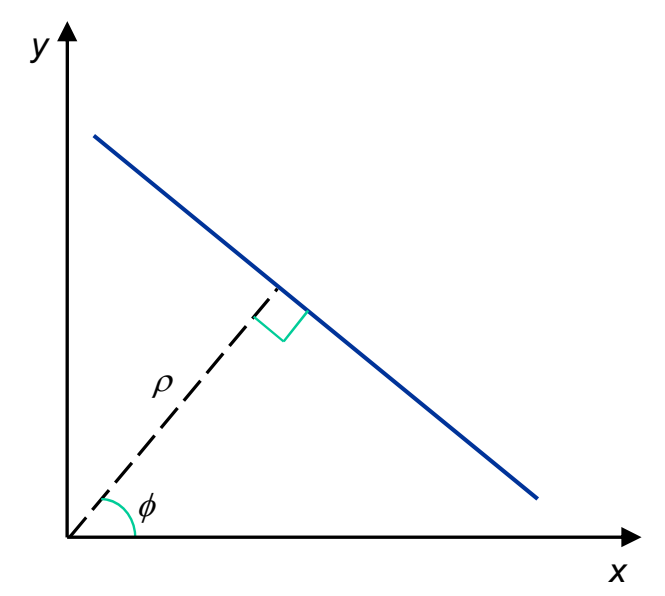

Fig. 8. $P$ and $\phi$ representation of a straight line.

\section{PERFORMANCE OF F-K TRANSFORM FOR TORPEDO DETECTION}

An experiment was conducted to study the performance of F-K transform for torpedo detection. Towed array data was collected during torpedo trial. The same array described in section 4 was used for the trial. Detection processing using conventional frequency domain beamformer with energy detector and F-K spectrum was attempted. It was observed that an average $6 \mathrm{~dB}$ SNR improvement at the detector output with F-K transform compared to conventional frequency domain beamformer with energy detector. Considerable reduction in beam width and side lobe levels was also observed with F-K processing. Reduced beam width and lower side lobe levels are essential features for accurate bearing estimation and discriminating nearby targets.

It is not practical to conduct trials with torpedo to measure SNR improvement at different towing speeds. The following method was used to extrapolate SNR improvement for torpedo detection at different towing speeds from the towed array data collected during the experiment described in section 4 . Torpedo signature was added with towed array sensor data collected at different towing speeds with selected SNR. Detection processing was done on this data using F-K transform and conventional frequency domain beamformer. Average SNR improvement observed at detector output for different towing speeds are tabulated in Table 2. Due to improved SNR at the detector output, early detection of torpedo threat is possible with F-K processing. This will enable the platform to initiate evasive actions and counter attacks at the earliest to escape from the torpedo threat.

Table 2. SNR improvement with F-K processing

\begin{tabular}{|c|c|c|}
\hline NO & $\begin{array}{c}\text { TOWING SPEED } \\
\text { (Knots) }\end{array}$ & $\begin{array}{c}\text { AVERAGE SNR } \\
\text { IMPROVEMENT AT THE } \\
\text { DETECTOR OUTPUT } \\
\text { WITH F-K PROCESSING } \\
(\mathrm{DB})\end{array}$ \\
\hline 1 & 6 & 1.0 \\
\hline 2 & 8 & 2.0 \\
\hline 3 & 12 & 3.5 \\
\hline 4 & 16 & 6.0 \\
\hline 5 & 18 & 8.0 \\
\hline
\end{tabular}

\section{CONCLUSION}

Frequency wavenumber transform for identification and removal of non-acoustic noise from towed array sonar in presented in this paper. Result of sea trial data analysis reveals that non-acoustic noise is a serious problem in towed arrays towed at higher towing speeds. Performance of towed arrays used in torpedo defence systems are seriously affected by this low frequency noise components. F-K transform is an effective tool for enhancing the detection capability at low frequency band by minimising the influence of non-acoustic 
noise in the detection output. Spatio-amplitude and spatiotemporal information generated form F-K transform is used for target detection, localisation and classification. Significant SNR improvement is observed at detector output with F-K transform compared to conventional frequency domain beamformer with energy detector. Long range detection of torpedoes are possible with improved SNR at the detector output. Reduced beam width and lower side lobe levels are also observed at the detector output. Automatic target detection using Hough Transform is also presented in this paper. This is an essential feature for torpedo detection. Adaptive noise cancellation techniques for the removal of non-acoustic noise from towed array sonars is the area of future research.

\section{REFERENCES}

1. Ramanarasaiah, N.K., Introduction to Torpedo Technology, DRDO Monogram Series-I, India, 1993.

2. RaviKumar, E. \& Rao, K. S., "Anti-Submarine / Ship Warfare (ASW): Tactical Assessment and Statistical System Analysis," International Conference on Systemises, Cybernetics and Informatics, p. 31-36, 2009.

3. Technologies for Sonar Systems, Technology Focus, DRDO Bulletin, vol. 18, No 4, 2010.

4. Waite, A.D., Sonar for Practising Engineers, John Wiley \& Sons Ltd, UK, 3rd Edition, 2002.

5. Krim, H. and Viberg, M., "Two Decades of Array Signal Processing Research.," IEEE Signal Processing Magazine, p. 67-94, July 1996.

6. Lasky, M., Doolittle, R.D., Simmons, B.D. and Lemon, S.G., "Recent progress in towed hydrophone array research," IEEE Journal of Oceanic Engineering, p. 374-387, vol. 29, no. 2, April 2004.

7. Haykin, S., Justice, J.H., Owsley, N.L., Yen, J.L. and Kak, A.C., S"onar array processing," Array signal processing, Prentice-Hall. 1985.

8. MENG, J., WANG, Y.J., CAI, L. and WEI, R., "Research on the Combination of Underwater Acoustic Countermeasure Equipments Against Torpedo," Proceedings of MATEC Web of Conferences, p. 13, 2016.

9. Chen, Y. and Qiu, Y., "Simulation-based effectiveness analysis of acoustic countermeasure for ship formation," 4th International Conference on Systems and Informatics (ICSAI), Hangzhou, p. 756-761, 2017.

10. Xiaoheng, D., Yiqi, Z., Minghang L. and Zhengyu, W., "The Surface Ship Torpedo Defense Simulation System," IEEE 3rd International Conference on Image, Vision and Computing (ICIVC), Chongqing, p. $802-806,2018$

11. AjithKumar, K., Jomon G. and Jagathyraj, V.P., "Requirements Analysis of an Integrated Sonar Suite for Surface Ships: Systems Engineering Perspective," Defence Science Journal, p. 366-370, 64(4), 2014.

12. Krishna, L.G. and Nandakumar, C.G., "Studies on Underwater Vibration Isolation Module". International Journal of Innovative Research in Science Engineering and Technology, p. 422-428, Volume 2, Special Issue 1, December 2013.

13. Wagstaff, R., "A computerized system for assessing towed array sonar functionality and detecting faults," IEEE Journal of Oceanic Engineering, p. 529-542, vol. 18, no. 4, 1993.

14. Maidanik G. and Jorgensen, D., "Boundary Wave-Vector Filters for the Study of the Pressure Field in a Turbulent Boundary Layer," The Journal of the Acoustical Society of America, p. 494-501, vol. 42, 1967.

15. Gopi, S., Felix, V.P., Sebastian, S., Pallayil, V. and Kuselan, S., "In-situ non-acoustic noise measurement system for towed hydrophone array," IEEE Instrumentation \& Measurement Technology Conference Proceedings, Austin, TX, p. 913-916, 2010.

16. Abshagen, J., Kuter, D. and Nejedl, V., "Flow-induced interior noise from a turbulent boundary layer of a towed body". Advances in Aircraft and Spacecraft Science, p. 259-269, 3(3), 2016.

17. Ketchman, J., "Vibration induced in towed linear underwater array cables," IEEE Journal of Oceanic Engineering, p. 77-87, vol. 6, no. 3, July 1981.
18. Beerens, S.P, Ijsselmuide, S.P., Volwerk, C., Trouve, E. and Doisy, Y., "Flow noise analysis of towed sonar arrays," UDT Europe. Nice, France, p. 392-397, 29 June - 1 July, 1999.

19. Blake, W., "Fundamental underwater noise sources," Encyclopaedia of Acoustics. M.J. Crocker, Editor, John Wiley \& Sons: New York, p. 501520, vol.1, 1997.

20. Knight, A., "Flow noise calculations for extended hydrophones in fluid and solid-filled towed arrays," Journal of the Acoustical Society of America, p. 245-251, Vol. 100, No. 1, 1996.

21. Unnikrishnan, K.C., Pallayil, V., Chitre, M.A. and Kuselan, S., "Estimated flow noise levels due to a thin line digital towed array," OCEANS 2011 IEEE - Spain, Santander, p. 1-4, 2011.

22. Ronald, A.M., "Comparison of efficient beamforming Algorithms," IEEE Transactions on acoustics, Speech and Signal Processing, ASSP32, p. 548-557, no. 3, June 1984.

23. Shefeng, Y. and Yuanliang, M., "High-resolution broadband beamforming and detection methods with real data". Acoust. Sci. \& Tech., p. 73-76, vol. 25, no. 1, 2004.

24. George J., Joseph V.J. and Santhanakrishnan, T., "MVDR beamformer with subband peak energy detector for detection and tracking of fast moving underwater targets using towed array sonars," Acta Scustica United with Acustica, p. 220 - 225, vol. 105, 2019,.

25. Liu, W., Huang, W., Wei, Y. and Chen, X., "A noise reduction method based on F-K transform for optical fiber hydrophone towed array," IEEE/OES China Ocean Acoustics (COA), Harbin, p. 1-5, 2016.

26. Maranda, B., "Efficient digital beamforming in the frequency domain," The Journal of the Acoustical Society of America, p. 1813-1819, vol. 86, 1989.

27. Suojoki, T. and Tabus, I., "A novel efficient normalization technique for sonar detection” Proc. Int. Symp. Underwater Technolog, p. 296-301, 2002.

28. Linda, G.S. and George, S., Computer Vision, Prentice-Hall, Inc. 2001. 29. Duda, R.O. and Hart, P.E., "Use of the Hough Transformation to Detect Lines and Curves in Pictures". Comm. ACM, p. 11-15, Vol. 15, January 1972 .

Mr Jomon George received his BTech (Electrical and Electronics Engineering) from Mahatma Gandhi University, Kerala, India and MTech (Electronics) from Cochin University, Kerala, India. Currently pursuing his $\mathrm{PhD}$ at Cochin University. $\mathrm{He}$ is working as Scientist at DRDO-Naval Physical and Oceanographic Laboratory, Kochi. His research interests include Sonar system design, Sonar signal processing and Towed array sonar systems. In the current study, he has carried out the basic research, system implementation, data analysis and writing original manuscript.

Mr. Baiju M Nair received MSc (Physics) from Loyola College, Madras University, Chennai, India, and MTech (RFDT) from IIT Delhi. He is working as Scientist at DRDO-Naval Physical and Oceanographic Laboratory, Kochi. His research interests include: Ocean Acoustic Signal Processing, Array Signal Processing and Reverberation studies. In the current study, she helped in software coding, data processing and preparation of figures.

Dr T. Santhanakrishnan received the MSc (Physics) from Madurai Kamaraj University, Madurai, India, MTech (Lasers and Electro-Optical Engineering) and $\mathrm{PhD}$ in applied optics from Anna University, Chennai. He is working as Scientist at DRDO-Naval Physical and Oceanographic Laboratory, Kochi. His research interests include Development of optoelectronic systems for under water applications, optical interferometry, fiber optic hydrophones, laser-based instrumentation, Big data analysis, sentiment analysis from tweets, polymer composites, underwater target detection and tracking, and thin film PZT sensors. In the current study, he helped in formulation of concept and research objectives, overseeing the research activity and critically reviewing the manuscript. 